\title{
Herboriser en art : Comment le végétal intègre les pratiques artistiques de François Morellet, Anne et Patrick Poirier et Bernard Moninot
}

\author{
To botanize in visual arts: How plants integrate artistic practises of \\ François Morellet, Anne \& Patrick Poirier and Bernard Moninot
}

\author{
Elisabeth Amblard ${ }^{1}$ \\ ${ }^{1}$ Artiste et maître de conférences en Arts Plastiques, Université Paris 1 - Panthéon-Sorbonne, Ecole des Arts de la \\ Sorbonne UFR 04
}

RÉSUMÉ. Parmi les artistes contemporains aux pratiques en lien avec les plantes, le présent article propose de s'attacher à ceux qui « herborisent ». Trois démarches artistiques seront interrogées afin de déterminer les spécificités des rapports directs, de prélèvements, de conservation et d'étude entretenus avec le végétal : trois moments des œuvres de François Morellet (Géometrees, 1983-1985), de Anne et Patrick Poirier (notamment les Herbiers, depuis 1971), de Bernard Moninot (La Mémoire du Vent, depuis 1997, Le pin peint, tout récemment).

ABSTRACT. Among the contemporary artists having a link with plants, the present article proposes to focus on those who « herborize ". Three artistic approaches will be investigated in order to determinate the specificities of the direct relationships, of sampling, of conservation and of study with the plant: three moments of the works of François Morellet (Géométrees, 1983-1985), of Anne and Patrick Poirier (notably the Herbiers, since 1971), of Bernard Moninot (La Mémoire du Vent, since 1997, Le pin peint, just recently).

MOTS-CLÉS. agentivité végétale, herborisation, geste, impermanence, relevé, botanique, système, inventaire, capture, dispositif, arts plastiques.

KEYWORDS. vegetal agentivity, herborization, gesture, impermanency, survey, botany, system, inventory, capture, artistic device, visual arts.

«Commencer par le presque rien : deux petites branches qui bougent dans le grand inconnu; Un grain de compréhension recueilli par un peu d'attention » ${ }^{1}$. Alexandre Hollan, 10.9. 2009

Parmi les artistes contemporains, nombreux, ayant un lien avec plantes, s'attacher à ceux qui herborisent est l'orientation première du présent propos. C'est pour leurs rapports directs, de prélèvements, de conservation et d'étude du végétal que trois démarches artistiques sont sélectionnées par un mélange d'intuition et d'hypothèses à vérifier : trois moments de l'œuvre de François Morellet ${ }^{2}$, d'Anne et Patrick Poirier ${ }^{3}$, de Bernard Moninot ${ }^{4}$. D'origines et d'horizons divers, ce ne sont pas des données historiennes qui pourront les associer simplement. C'est bien davantage par leur singularité, quasi événementielle, que seront saisies leurs approches, portées par un esprit de recherche et d'émulation curieuse. Aussi suivra le récit de trois rencontres cherchant plus à diversifier les expériences qu'à les unifier. Sur chacune de ces pratiques, se risquer à poser un regard mi-informé, mi-naïf et commencer par la plus improbable : celle de François Morellet.

\footnotetext{
${ }^{1}$ Alexandre Hollan : Je suis ce que je vois, Notes sur la peinture et le dessin, 2006-2011, Le Temps qu'il fait, 2013, p. 14. A. Hollan (né en 1933), artiste d'origine hongroise, vivant et travaillant en France depuis 1956.

${ }^{2}$ François Morellet (1926-2016), artiste français.

${ }^{3}$ Anne Poirier (née en 1941) et Patrick Poirier (né en 1942), couple d'artistes français.

${ }^{4}$ Bernard Moninot (né en 1949), artiste français. 


\section{François Morellet : la nature ordinaire au regard de la géométrie}

François Morellet revendique une approche minimale et géométrique de l'art : il souhaite être objectif, réduire au maximum les interventions subjectives de l'artiste, se tenir loin de l'idée de nature ${ }^{5}$. En cela, a priori, il se situe aux antipodes de ce qui nous concerne ici. Il privilégie les logiques internes de l'œuvre, faisant système, ce qui en revanche, le rapproche de considérations scientifiques. "Une expérience véritable doit être menée à partir d'éléments contrôlables, en progressant systématiquement suivant un programme. $»^{6}$ dit F. Morellet.

Il est intriguant et certainement non anodin de voir apparaitre des fragments de nature un jour de 1983, au moment où F. Morellet débute une nouvelle série, nommée Géométree, alliance insolite, comme l'indique son nom, de la géométrie et de l'arbre, profitant et jouant d'homophonie francoanglaise. Le point de départ de chacune de ces auvres est une herbe, ou une brindille, le plus souvent une branche, un élément prélevé à l'extérieur, libre, loin de tout contrôle, manifestation de la nature végétale. "Je t'entrevois flânant "utilement" dans le jardin de Cholet, avisant la brindille ici-bas chue [...] » écrit Mario Toran à son ami F. Morellet ${ }^{7}$. Au-dehors, l'artiste glane, ci et là quelques branchages et autres tiges végétales, la part première de ses œuvres. Il pioche dans ce qui est à portée de main, simples «étant là » naturels - qui constitueront la base de son travail. Ce simple geste - qui conjugue néanmoins à la fois le hasard d'une rencontre, un prélèvement et un choix - sera incorporé à un processus car c'est la structure même du végétal qui définira la construction de l'œuvre.

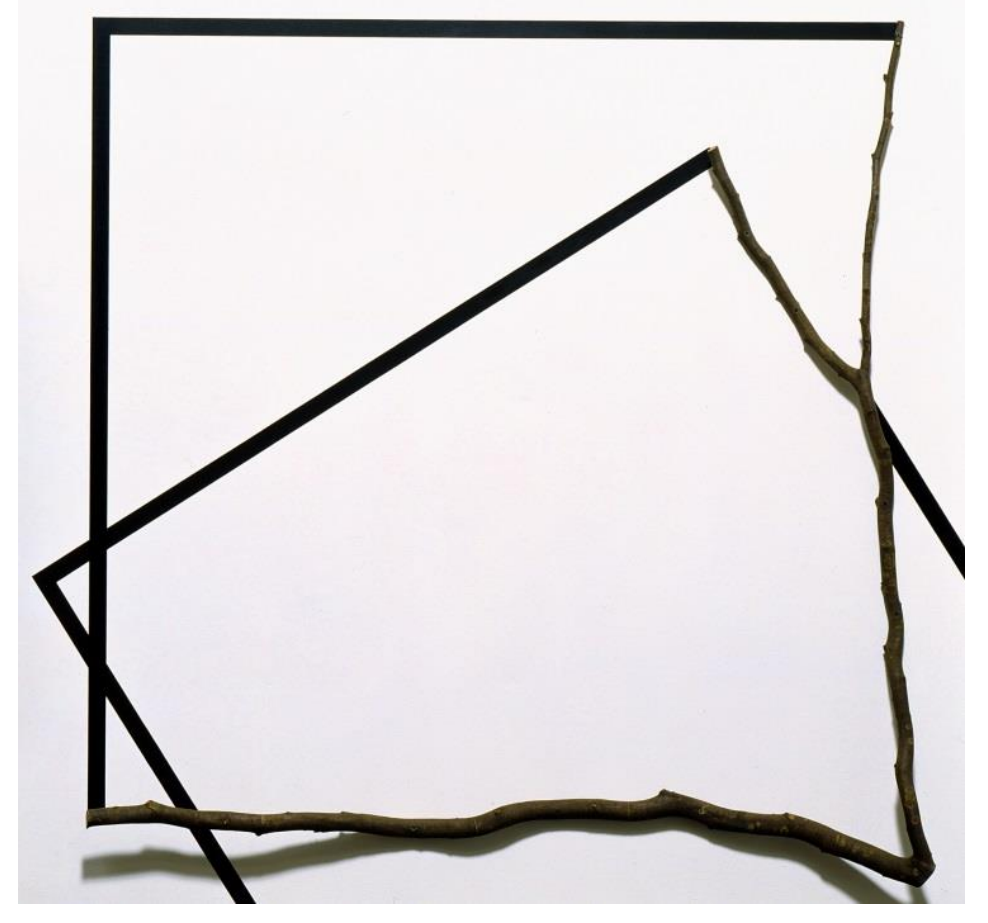

Figure 1. François Morellet, Géométree $n^{\circ} 109$ (Parjure $\left.n^{\circ}{ }^{1}\right), 1993$

branche et acrylique sur bois, $240 \times 240 \mathrm{~cm}$

collection les Abattoirs Musée - Frac Occitanie Toulouse

(C) ADAGP, Paris ; photo: Grand Rond Production

\footnotetext{
${ }^{5}$ Voir F. Morellet, "Les années soixante-dix », Art Actuel, Genève, Skira, n6, 1980, p. 95 : « Si depuis 1950 mes œuvres flirtent avec le vide, $c^{\prime}$ est avec cette espèce bien particulière de vide dû à l'absence de "nature". "

${ }^{6}$ F. Morellet, GRAV, Plaquette de l'exposition, galerie Denise René, Paris, 1962.

${ }^{7}$ Lettre de Mario Toran à François Morellet, Les Sables-d'Olonne, samedi 26.X.85, François Morellet, Géométree, catalogue de l'exposition, Abbaye Royale de Fontevraud, 30 novembre 1985-18 janvier 1986, Fonds Régional d'Art Contemporain des Pays de la Loire, p. 26. Mario Toran : auteur, commissaire d'exposition, 1949-1990. 
Géométree $n^{\circ} 109$ (Parjure $\left.n^{\circ} 1\right)^{8}$, de 1993 en est un exemple. La branche choisie, par une première ramification, forme un « $\mathrm{V} »$ qui sera un angle d'origine, placé en bas à droite, en coïncidence avec celui du panneau de bois peint en blanc. Elle est donnée brute mais orientée de façon à définir deux côtés primaires d'un carré quand deux bandes noires rectilignes - et parallèles aux bords du support -, tracées à la peinture acrylique, le complètent avec une rigueur toute mathématique. Se dessine une figure intégrale, droite, centrale et composite.

La ramification verticale se dédouble en une nouvelle branche secondaire ${ }^{9}$ et donne le départ d'un nouveau «carré » de même mesure, placé en diagonale, en partie hors-champ, lui aussi orienté à partir de ce segment naturel, complété par des bandes noires d'identique épaisseur. Géométree $n^{\circ} 109$ est assez complexe, mêlant et démêlant ce qui est du tracé de ce qui est « naturel », réel. Il est à noter d'ailleurs que la photographie « officielle » souligne l'ombre portée de la branche, et ourle ce qui fait tableau d'un relief entrant dans la réalité du lieu qui l'expose.

Il y a beaucoup d'économies dans cette pièce. Tout d'abord celles préexistantes à la série comme «... le choix du noir et du blanc ... car c'est les deux couleurs que tu peux définir d'une façon objective : le blanc réfléchit le plus la lumière, et le noir l'absorbe. Et si je prends un carré, c'est parce qu'il se définit d'une manière très simple, par une dimension. ${ }^{10}$ Une part de la ligne de conduite de l'artiste est maintenue; une autre est pourtant aussi volontairement perturbée. «Géométree, la buissonnière, inaugure les dimanches de l'œuvre de Morellet. » ${ }^{11}$ En effet, depuis le milieu des années cinquante, F. Morellet fait œuvre systématique, programmée avant l'exécution, usant d'une technique impersonnelle. Ici elle incorpore une dépendance, s'infléchit en fonction de ce qui est trouvé. L'insertion volontaire d'un élément extérieur, premier et décisif formellement, provoque une certaine agitation. Car il semble maintenant que ce sont les branches mêmes, choisies par l'artiste, qui induisent le programme de construction du tableau. Dans sa volonté générale de réduire au minimum son intervention, il accorde une part décisive au végétal. Il est l'amont de la réalisation. L'œuvre ne se situe plus dans l'autoréférentialité d'un système qui génère des figures en développement autonome, indépendamment de l'artiste. Le végétal trouvé met à sa disposition une incitation. Il propose. L'artiste le relaie, décide d'un plan d'action, qui est un plan de peintre et de sculpteur tout à la fois. Une simplicité n'exclut pas une réelle complexité. La géométrie analytique couvre de ses logiques le fragment glané. Ainsi se formule l'hypothèse : une fois reconnue, une parenté possible se synthétise en une écriture linéaire (crayon gras, acrylique, marker, fusain, peinture ...) formant figure. Dialogue instauré entre la nature et une idée mathématique. Le végétal est analysé ; il contrecarre (momentanément) les positions antérieures de F. Morellet. L'œuvre s'élabore à partir de l'objet « ramassé » combiné au regard que l'artiste porte sur lui. Ce regard est une étude détaillée qui cherche la juste orientation et l'articulation. Des prolongements géométriques correspondent aux amorces naturelles, départs de branches, mesure de segments, élan d'une courbe, continuité en arc de cercle, angle formé par un embranchement qui, dépeint, en miroir, produira un carré ou toute autre figure géométrique simple. Élément saillant du panneau, de caractère botanique, marquant un intérêt pour une forme ordinaire, objet trouvé, le végétal se trouve greffé à un homologue mathématique. Associés l'un à l'autre, l'un naturel, singulier, réel, l'autre, pendant abstrait, général et idéel, selon les cas, simplifient la forme, la complètent, lui trouvent une

\footnotetext{
${ }^{8}$ Cent-une œuvres représentent la totalité des Géométrees (cent-trois moins deux nombres non-attribués (41 et 42 ). En 1985 F. Morellet décide d'arrêter cette série. Néanmoins il se « laisse quand même la possibilité pour les cadeaux de fin d'année ou de mariage, d'aller jusqu'au $n^{\circ} 110$ (mais pas plus c'est promis) ». P. 92. Ce qui explique le présent intitulé : Géométree $n^{\circ} 109$ (Parjure $\left.n^{\circ} 1\right), 1993$, branche et acrylique sur bois, $240 \times 240 \mathrm{~cm}$, collection les Abattoirs Musée - Frac Occitanie Toulouse / (C) Adagp, Paris ; photo : Grand Rond Production.

${ }^{9}$ (Ce qui n'est pas tout à fait vrai physiologiquement).

${ }^{10}$ Images de la culture : François Morellet, entretien avec Daniel Soutif, 1999, documentaire, 52 min, couleur, réalisation : Camille Guichard.

${ }^{11}$ Lucie Normandin, "Géométree ou La buissonnière du Système-Morellet », Vie des arts, volume 30, n 119, Montréal, juin-été 
correspondance mentale. Ils procèdent d'un schème, principe général auquel correspondrait la forme naturelle et particulière. Modestement la nature dicte, contrainte à son épure, une continuation. La nature est présentée - plus que représentée. La nature est synthétisée sous forme géométrique. Les liens sont opérants.

À cet endroit est à noter que, dans chaque Géométree, l'instantanéité de la forme géométrique, par l'existence même du végétal, présente une lisibilité du tracé et intègre le principe de croissance des branchages, pour un développement continué, mesuré, poursuivant de manière méthodique le commencement végétal. Néanmoins, le mode objectif de l'ensemble est appuyé. Il ne s'agit pas là de facture personnelle ou sensible. Dans l'écriture déposée tout est réalisation géométrique, quasi mécanique, envers, une fois encore, du devenir organique végétal.

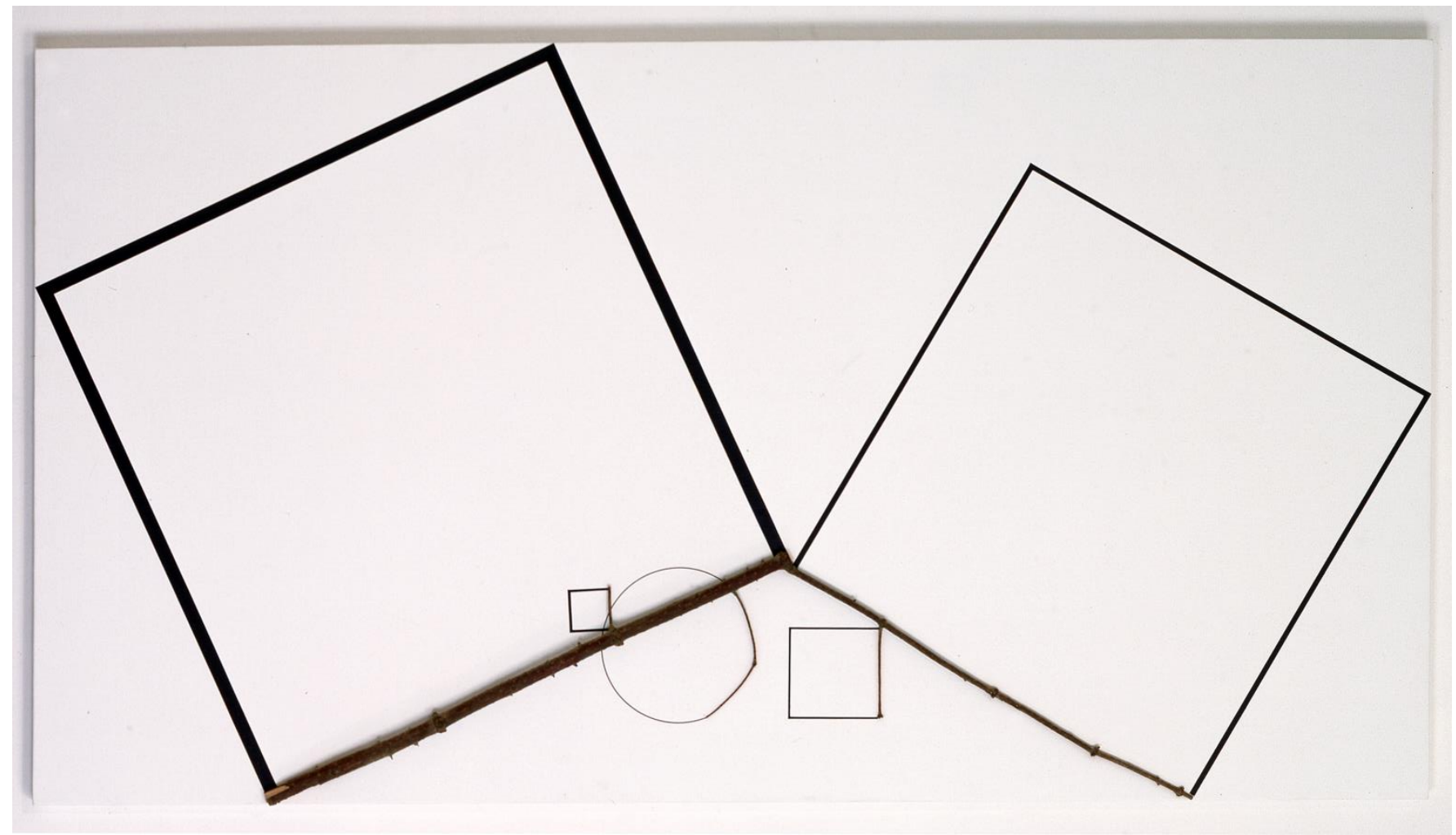

Figure 2. François Morellet, Géométree n`86, 1984

acrylique blanche et bois sur toile, $120 \times 183 \mathrm{~cm}$

Collection du Cnap (C) ADAGP, Paris, CNAP

Participant d'une vision également analytique, en son aval, les Géométrees seront possiblement classés. Ce moment est même fondamental pour comprendre la démarche de l'artiste quand « le classement est l'action de ranger effectivement d'après un certain ordre [...] [et] la classification est l'ensemble des règles qui doivent présider au classement effectif ou qui déterminent idéalement un ordre dans les objets $\gg$. François Morellet en affirme l'établissement selon une logique qui lui appartient. Il écrit : «toujours les classements que je trouve satisfaisants ressemblent un peu à cette merveilleuse énumération inventée par Borgès [...] où les animaux sont divisés en a) appartenant à l'Empereur b) embaumés, c) apprivoisés, d) cochons de lait, e) sirène, f) fabuleux, etc... ${ }^{13}$. Néanmoins il poursuit et précise :

« Nous avons cependant, à contrecœur, abandonné la tentation d'être satisfaisant pour la prétention d'être logique.

\footnotetext{
${ }^{12}$ Le Littré, Dictionnaire de la Langue Française, par Émile Littré, 1873, Tome 1, p. 639.

${ }^{13}$ François Morellet, Géométree, op. cit., p. 4. 
Et un classement logique se doit d'être aveugle sur toutes les qualités des éléments à classer sauf une. Cette qualité a été : l'aspect formel (avec entre parenthèses mon système d'intervention

avec lignes (prolongées)

avec formes (fermées)

avec trames (complétées)

avec perpendiculaires (indiquées)

avec symétries (suggérées)

avec classification (impossible dans ce classement).

Ce classement est bien sûr arbitraire. »

F. Morellet insiste sur la logique de répartition formelle des catégories qui est elle-même un axe possible parmi d'autres. D'ailleurs, après ces mots, l'artiste propose d'autres classements alternatifs pour marquer les ouvertures voire les porosités. L'enjeu est contraire au raidissement, souhaite et sollicite la variabilité du dispositif général. Ce qui interpelle là, sans surprendre, est la nécessité d'opérer des regroupements qui disent combien tous les Géométrees, par-delà leur titre, relèvent à la fois d'un grand ensemble, mais aussi des subdivisions, d'une attitude raisonnée et relevant d'une cohérence dans la variété.

Via l'intégration du végétal, F. Morellet se détourne de l'autoréférentialité des systèmes jusqu'alors mis en place, pour s'adonner quelques temps à l'élaboration d'un autre principe, inclusif, impliquant le choix et portant un regard attentif à ce qui émerge de la nature sous des formes simples pour en donner lecture. Double sens d'évocation, en rien réducteur - plutôt contrastant - de la nature vers la géométrie et réciproquement. L'une et l'autre, suivant leur composante structurelle, se répondent systématiquement. Association des contraires, en tout état, interrelation de provenances distinctes, elles travaillent à établir des conjonctions, jouent de prolongement, de ramification. Leur jointure propose la formation de schèmes représentatifs, en une liaison covalente. De manière identique à la nature, la géométrie procède alors du branchement (en français littéraire, "pousse des branches» du végétal), développant des rapports nouveaux de coïncidence et de coexistence. Elle se donne à penser comme géométrie non plus minimaliste mais comme géométrie relative, ambiante, inclusive.

Enfin, à la fin du catalogue dédié aux Géométrees, F. Morellet introduit deux visuels de peintures $^{14}$ de 1949 «branche éloignée de la famille, une branche pas encore entrée dans le système » pour en dire avec une légèreté assumée : «Ainsi, une ancienne période un peu honteuse profite souvent d'une parenté plus ou moins établie avec une plus jeune pour apparaitre enfin (36 ans après) sur un catalogue ${ }^{15}$ tel un aveu jovial, consommé et nécessaire.

\section{Anne et Patrick Poirier : le végétal, signe impermanent d'un lieu et d'une mémoire}

S'ils sont loin de constituer la totalité de l'œuvre d'Anne et Patrick Poirier, les herbiers, dans leurs distinctes variations au cours du temps, en constituent tout un pan. Aussi les artistes, de but en

\footnotetext{
${ }^{14}$ Branches et graines, 1949, huile sur contreplaqué, 34 x $59 \mathrm{~cm}$ et Herbes, 1949, peinture à la colle sur une planche, 23 x $68 \mathrm{~cm}$.

${ }^{15}$ François Morellet, Géométree, op.cit., p. 90. 
blanc, énoncent que le rapport au végétal est «une constante dans leur travail » ${ }^{16}$. Déjà, l'un et l'autre, dans les campagnes nantaise et provençale, enfants ${ }^{17}$, collectionnent «les petites choses de la nature ».

De 1967 à 1971, A. et P. Poirier sont pensionnaires de la Villa Médicis à Rome, de la Villa et de son vaste jardin - qui entretient encore aujourd'hui, en grande partie, l'ordonnance qui l'aménage au $\mathrm{XVI}^{\text {ème }}$ siècle, à l'image des jardins botaniques toscans. Ils se souviennent y avoir ramassé, aux pieds des haies de lauriers taillées en de hautes silhouettes régulières, formant des chambres, des feuilles ${ }^{18}$ un peu noirâtres, déjà signe des pollutions alentours. "La nature souffrait de la proximité d'une espèce d'autoroute, les hydrocarbures se déposaient ». Contemporains de l'œuvre Ostia Antica, 1969-1972 ${ }^{19}$, naissent alors les premiers herbiers ${ }^{20}$ des artistes, qui ont l' « œil à ce qui traine au sol, sans idée préconçue », comme pour Herbier ${ }^{21}$, 1971, assemblage sur fond en papier dans un emboîtage formé d'un sous-verre et d'un cadre en bois, qui rappelle les boites des entomologistes, piquées de papillons ou autres insectes. Il porte, manuscrite, la mention descriptive de l'œuvre : « 3 feuilles de lauriers cassées en 3 morceaux et présentées conservées ».

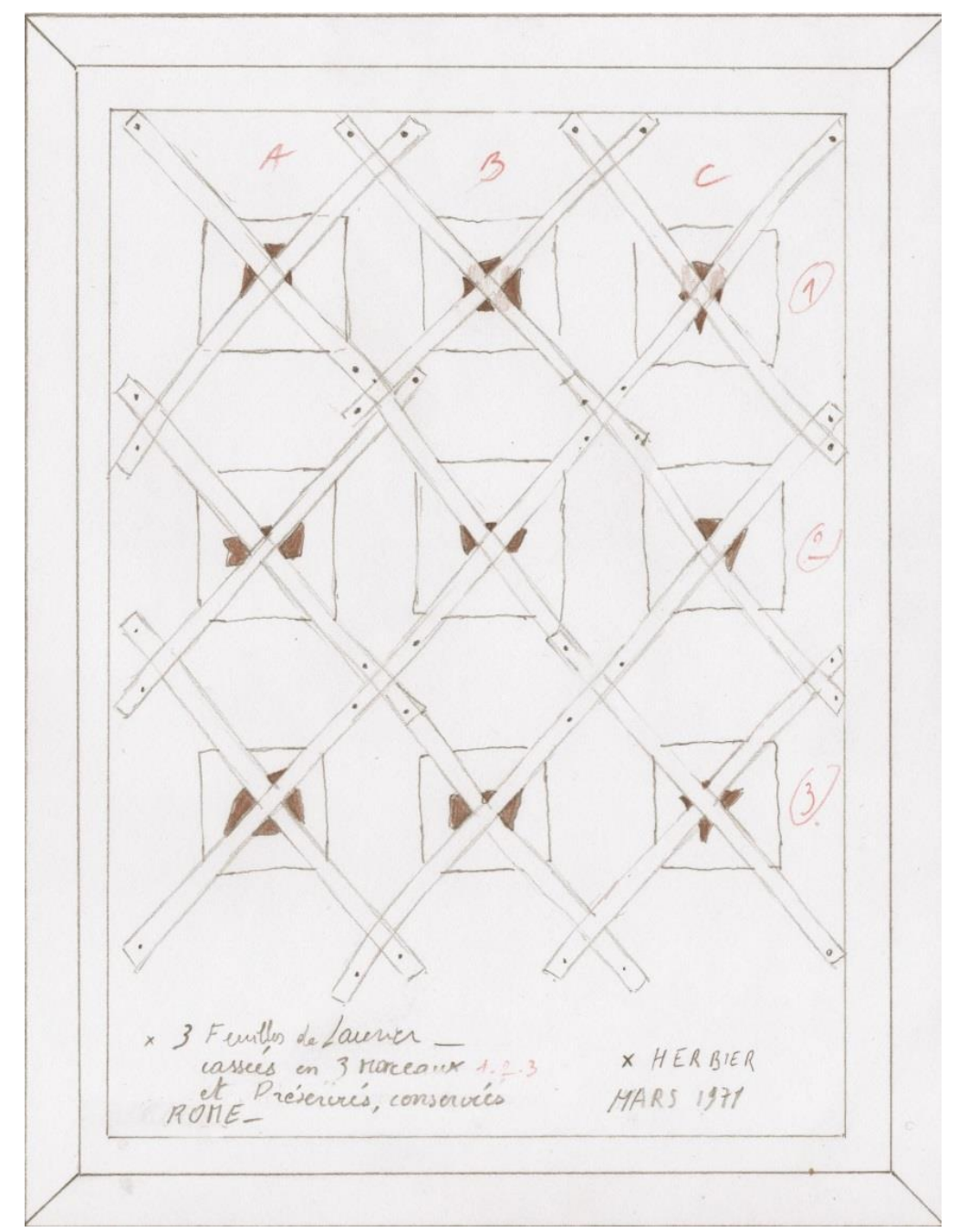

Figure 3. A. et $P$. Poirier, Herbier, 1971, $42 \times 32 \mathrm{~cm}$, collection particulière (extrait de l'interprétation graphique de 5 herbiers avec l'autorisation de Anne et Patrick Poirier / ADAGP)

\footnotetext{
${ }^{16}$ Entretien du 9 janvier 2021 - les guillemets de la présente partie consacrée à Anne et Patrick Poirier font référence à cet entretien.

${ }^{17}$ Anne Poirier, née à Marseille en 1941, Patrick Poirier, né à Nantes en 1942.

${ }^{18}$ Que l'on sait pourtant coriaces, persistantes et parfumées.

${ }^{19}$ Ostia Antica, 1969-1972, terre cuite, $12 \times 6 \mathrm{~m}$, maquette des ruines du port antique de Rome reconstruites brique à brique.

${ }^{20}$ "On a fait beaucoup d'herbiers dans ces années-là. On appelait ça les journaux d'archéologues. "

${ }^{21}$ A. et $P$. Poirier, Herbier, 1971, $42 \times 32 \mathrm{~cm}$, collection particulière. 
Les feuilles de lauriers séchées, déposées sur des carrés de coton, sont maintenues par des bandelettes très fines de papier Japon formant croix, épinglées. On imagine le soin accordé à la suite des gestes, méthodiques, nécessaires à cette présentation. Après une collecte, les artistes conçoivent les conditions de sa conservation et renseignent celle-ci, par le détail, en des annotations multiples faisant repères. Titré et daté en bas à droite «Herbier », « mars 1971 » et situé à « Rome », en bas à gauche, l'espace entier de la boîte se subdivise en trois lignes sur trois colonnes chiffrées au crayon rouge « $\mathrm{A}, \mathrm{B}, \mathrm{C}$ » en haut de gauche à droite, «1,2,3 » sur le côté droit de haut en bas. Chaque partie de ce quadrillage reçoit un des neuf fragments de feuilles. L'œuvre présente toutes les qualités des exsicatta ${ }^{22}$.

Anne Poirier : «L'une des autres pratiques légères auxquelles on se livrait sur les sites archéologiques, c'était l'herborisation. Il s'agissait de la tenue de carnets qu'on appelait nos Carnets de fouilles. Cela consistait à prendre de la végétation et en faire des livres. On les collait, on écrivait des choses par-dessus, on faisait des dessins. Par exemple, si on prenait une feuille, on faisait le dessin à côté de la feuille en sachant que le temps allait altérer le vrai végétal et qu'ensuite seul le dessin resterait comme témoin. On se livrait à une espèce de petit travail de lutte contre le temps, à la manière des archéologues qui font des inventaires, qui font des listes, qui relèvent des traces, qui relèvent des plans, qui prennent des photos, puis on ramenait tout ce matériel dans nos ateliers. $»^{23}$

Tout de la démarche herboriste du binôme se concentre en ces quelques phrases : les emprunts définitifs aux sites, leurs qualités de relevés, la légère résistance et la fragilité du végétal ${ }^{24}$, l'accompagnement des notes, dessins et photographies. Cela désigne leur « intérêt pour la nature, l'explorer, la voir différemment» et notamment la manière de s'en saisir par un geste de prélèvement. Ainsi ils soustraient au lieu pour présenter au regard. Ils opèrent par captation au sens premier, en s'emparant physiquement de quelque chose jusqu'alors enraciné, accédant à un souvenir matériel de caractère métonymique désignant potentiellement le tout par la partie présentée, rendant possible son déplacement, sa mémoire ${ }^{25}$.

\footnotetext{
22 Exsicatta : spécimens desséchés de plante conservés dans un herbier.

${ }^{23}$ " Fragilité et esthétique de la ruine »-Conférence de Anne et Patrick Poirier - 29 avril 2010 - École Supérieure d’Art d’Avignon (ESAA) - Avignon - Présentation et modération : Gilles Coudert.

24 - « (...) la fragilité est notre guide depuis quarante-cinq ans » "Entretien d'Anne et Patrick Poirier », Mémoire à l'œuvre, dir. Gilles Couderc, Véronique Mori, Paris : A.P.R.E.S. Éditions, 2014, p. 49.

${ }^{25}$ À noter : l'exposition au Domaine de Chaumont, 31 mars - 4 novembre 2018, à la Galerie Basse du Fenil s'intitule “Herbarium Memoriae" que l'on traduit par « Herbier de la mémoire ». 


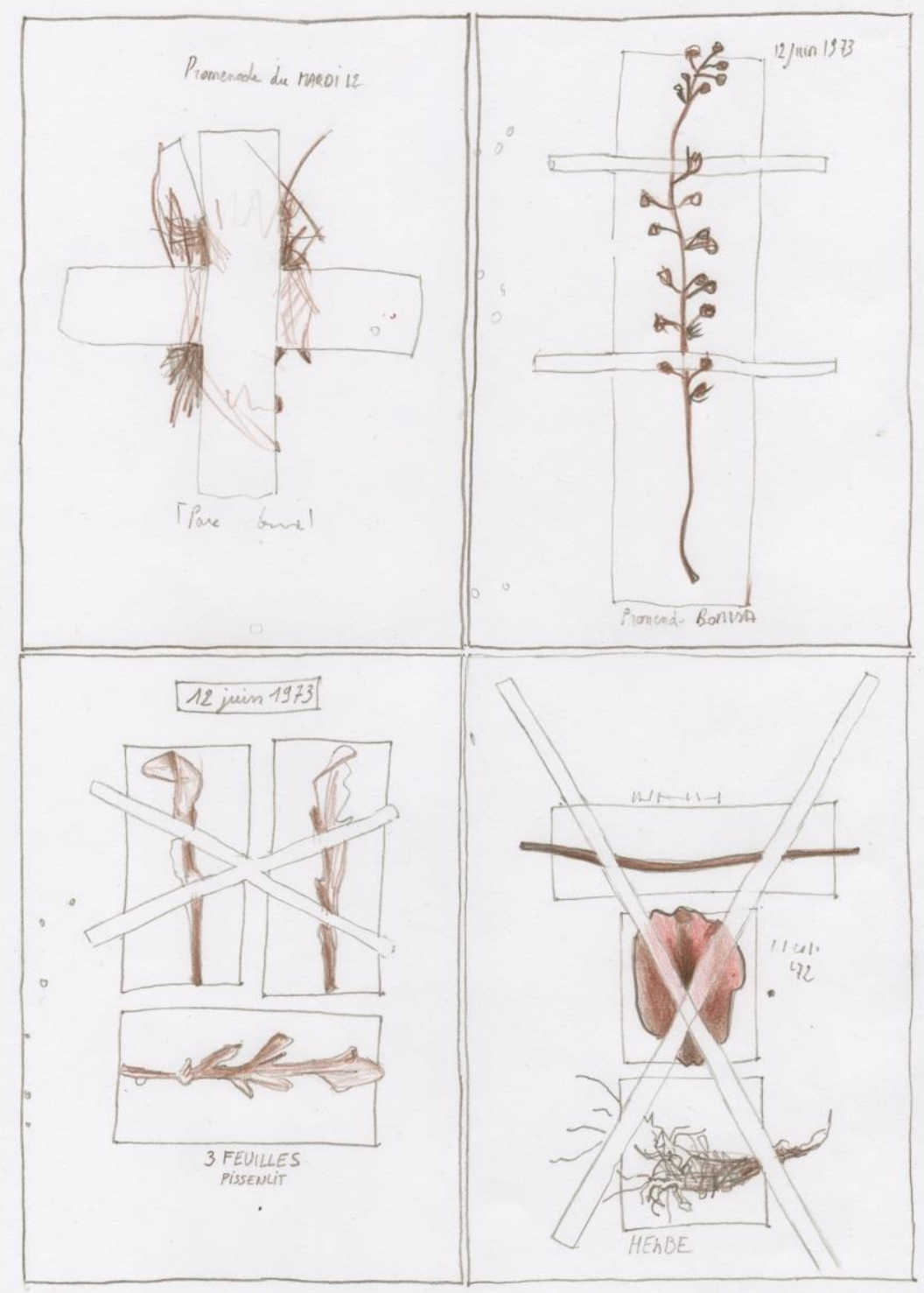

Figure 4. A. et P. Poirier, Journal herbier de Bordeaux, 1973, collection particulière, 4 feuillets du mardi 12 juin 1973

(extrait de l'interprétation graphique de 5 herbiers avec l'autorisation de Anne et Patrick Poirier / ADAGP)

Le Journal herbier de Bordeaux ${ }^{26}$ procède d'une même pratique, de mai à juillet 1973. Ensemble ils mènent une investigation directe, sur le terrain. C'est en marchant, qu'A. et P. Poirier se documentent sur le lieu systématiquement, "sans le côté scientifique, de manière différente » précisent-ils. Pendant trois mois, les artistes sortent tous les jours et sillonnent la ville, pour la comprendre. Durant le temps de leur résidence, ils inventorient en une suite de 3500 petits feuillets, les fruits, plus ou moins nombreux, d'une collecte quotidienne. Sont ainsi répertoriées les plantes autochtones - et autres mousses qui souvent échappent à la vue - modestes, récoltées en arpentant les rues de Bordeaux. De retour, ils notent les dates, les heures, les noms des plantes cueillies entre deux pavés, au bord d'un trottoir. «Pour tout conserver» ils trempent leurs spécimens dans la cire d'abeille, chaude, vierge, odorante, persistante ${ }^{27}$. Ainsi forment-ils une image indicielle du lieu, d'un réel - tangible - dont ils se saisissent par fragments, s'attachant à sa part végétale pour partie résistante aux aléas du temps. Ne dirait-on pas que cette restitution a à voir avec une forme de reconstitution? Les «herbes », sont les preuves d'un passage, d'un " hic et nunc », singulières, connaissances concrètes du lieu, par le menu.

\footnotetext{
${ }^{26}$ Journal herbier de Bordeaux, 1973, 3500 petits feuillets, 20 x $14 \mathrm{~cm}$ chacun, herbes sèches et collage.

${ }^{27}$ Pratique conservatoire toujours actuelle, continuée depuis lors. 
Premièrement présentées formant un énorme socle au sol donnant à penser à un livre manuscrit, les feuillets seront réunis par journée en de grands formats ${ }^{28}$, établissant chacun une carte du jour, une mémoire du lieu et de son expérience.

En 1977, A. et P. Poirier séjournent quelques mois à Berlin et commencent à faire des photographies à la chambre. Cette série de photographies est pour eux une « manière de prendre du recul, de se rappeler le paysage ». Ils se souviennent de la photographie d'un pétale de rose ramassé, de l'épine de la fleur avec laquelle ils gravent sur le pétale frais qui, très vite, se racornit. C'est dans une logique sans discontinuité de la pratique photographique qu'est fait Hommage à Blaschka ${ }^{29}$ Iris Violet, 2012. Le titre semble énigmatique. Renverrait-il à une ville de l'Est de l'Europe, au nom d'une femme ? Ce n'est pas la seule œuvre à contenir cet acte de reconnaissance, «Blaschka ». La première à intégrer cette dimension, On Extreme Fragility (Homage to Blaschka) ${ }^{30}$, date de 2003. C'est une œuvre en verre, sculptée à chaud dans la masse, présentant trois pétales, trois étamines (aux anthères ${ }^{31}$ brunes) et un pistil (portant stigmate) de lys, gigantesques et infiniment détaillés, comme tombés, sans se briser, au sol. La clé est là. Elle nous met sur une piste mêlant art et botanique. Elle renvoie à l'incroyable collection Ware ${ }^{32}$ conçue, détenue et exposée par le Harvard Museum of Natural History de Cambridge (Massachusetts). Véritable patrimoine scientifique, celleci, initiée par le professeur George Lincoln Goodale ${ }^{33}$, fondateur du Musée botanique de l'Université de Harvard, se compose de spécimens botaniques en verre de huit-cent-quarante-sept espèces végétales grandeur nature. Plus de quatre-mille-trois-cents modèles en présentent fidèlement chaque aspect: des coupes anatomiques, des parties agrandies, formant un incroyable corpus pédagogique, accessible à tous moments ${ }^{34}$, assurant leur étude, leur transmission. Fascinantes, elles relèvent de la virtuosité de Léopold Blaschka et de son fils Rudolph Blaschka, artisans verriers allemands $^{35}$ qui les produiront en cinquante ans, de 1886 à 1936. Leur vraisemblance est à ce point poussée qu'elle réduit la distinction entre objets artificiels et formes naturelles.

Ainsi donc, la photographie Hommage à Blaschka résonne comme le verre, celui façonné et teinté par les Blaschka, père et fils, qui puisent dans les qualités de leur matériau la grande solidité des couleurs et la tenue impeccable de leurs modèles en verre (face au temps).

La photographie Cibachrome ${ }^{36}$ répond à pareille problématique. Elle n'a nulle semblable pour capter et rendre la lumière, les couleurs naturelles, vraies, présentes à « la prise de vue ». Elle permet de travailler directement à partir d'une diapositive que les artistes constituent, disposant les

\footnotetext{
28 Journal herbier de Bordeaux - mardi 12 juin 1973, 1973, collage de 25 feuillets, $105 \times 77 \mathrm{~cm}$. Journal herbier de Bordeaux - dimanche 17, lundi 18 juin 1973, 1973, collage de 25 feuillets, $119 \times 78 \mathrm{~cm}$.

${ }^{29}$ A. et P. Poirier, Hommage à Blaschka - Iris Violet, 2012, $224 \times 151 \mathrm{~cm}$, édition unique, photographie, Cibachrome, Galerie Mitterrand.

${ }^{30}$ A. et P. Poirier, On Extreme Fragility (Homage to Blaschka), 2003, verre soufflé, dimensions variables, pétale le plus grand: 20,3 x 90,2 x 34,9 cm, The Corning Museum of Glass, État de New York. Assistant : Pino Signoretto, (1944-2017), Assistant, Vetreria Pino Signoretto, Studio (Italie, Venise, Murano). L'œuvre porte deux inscriptions au milieu de deux des pétales : FRAGILITY et HEART BEAT.

${ }^{31}$ Partie terminale de l'étamine, chargée de pollen.

${ }^{32}$ Du nom de Mme Elizabeth C. Ware et sa fille Mary Lee Ware, mécènes de la collection donnée à I'Université de Harvard en mémoire de leur mari et père Dr Charles Eliot Ware (1814-1887).

${ }^{33}$ George Lincoln Goodale (1839-1923), botaniste américain.

${ }^{34}$ La première destination de ces pièces est l'enseignement. George Lincoln Goodale peut les présenter à ses étudiants lors des cours et démonstrations.

${ }^{35}$ L'atelier des Blaschka était situé à Hosterwitz, près de Dresde, en Allemagne.

${ }^{36}$ Procédé qui permet d'agrandir des diapositives sur papier, aujourd'hui disparu depuis l'arrêt de la production du papier Cibachrome/Ilfochrome, au début des années 2010, seul fabriquant/fournisseur de ce papier lancé en 1963 (à noter : papier qui n'en est pas vraiment un puisque les tirages s'effectuent sur une base de polyester acétate stable, base plastique). 
pétales d'un iris entre deux plaques de verre à l'instar d'un biologiste préparant, dans son laboratoire, les lamelles pour une étude microscopique.

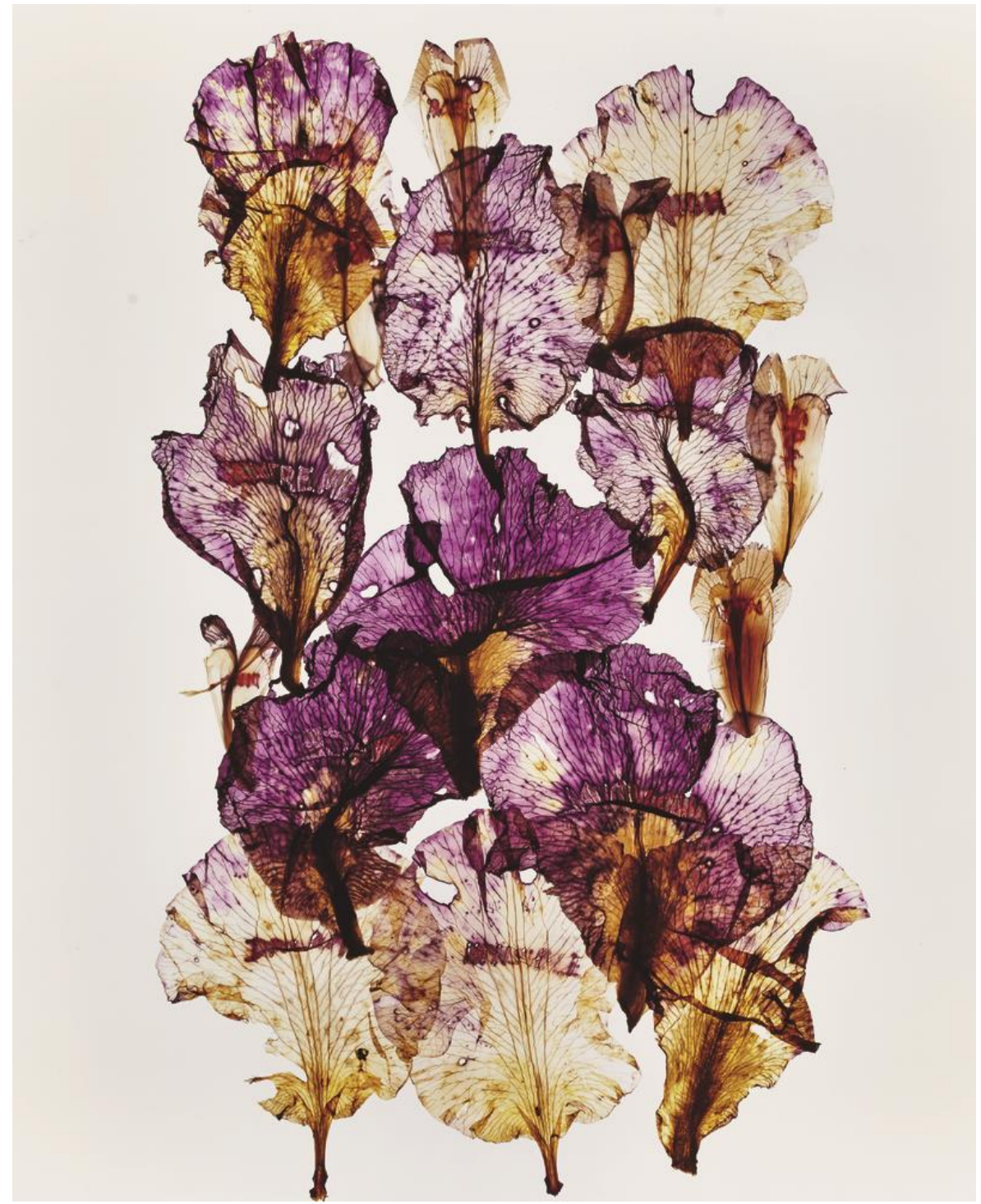

Figure 5. A. et P. Poirier, Hommage à Blaschka - Iris Violet, 2012, 224 x $151 \mathrm{~cm}$ (c) Anne et Patrick Poirier, Courtesy Galerie Mitterrand, Photo : J.C. Lett

En Provence, racontent Anne et Patrick Poirier ${ }^{37}$, au printemps (fin avril, mi-mai), les iris poussent en masse. Iris, fleurs d'un jour. Leurs pétales forment un «matériau incroyable»: complètement rigide à l'épanouissement de la fleur ; pourtant il s'abime très vite - en moins de deux heures - tant et si bien qu'il ne peut être manipulé. Sans résistance, il incarne la fragilité même. D'abord les pétales prélevés sont tatoués. Par le tatouage, la chair des choses est atteinte. A. et P. Poirier ne se contentent pas d'en rester à leur surface, ils inscrivent un langage ténu, messager, griffé, à même des pétales, marqués, à jamais scarifiés de mots au seuil de la lisibilité. Leur écorchure laisse lire «INVISIBLE», «EXTREME», quand d'autres écritures se perdent en évocation. Ensuite «il faut faire vite» pour surprendre la translucidité des végétaux directement saisis entre les lamelles d'une diapositive. Les pétales, réchauffés par la lumière, exhalent leur humidité, embuent les vitres et accélèrent leur dégradation. Ainsi à chaque «lame » correspondra un tirage unique, stoppant le cours du temps. La photographie n'est pas choisie pour sa capacité à démultiplier l'image, elle s'attache à saisir l'éphémère de ce qu'il est possible de voir; elle capte le seul instant. Aussi, elle évoque la floraison passagère, sa fragilité constitutive, ses variations. 
Car finalement c'est cela qui compte, c'est cela qui perdure. Les pétales sont la matérialité de l'instant.

Agrandis et fixés par le tirage photographique comme par la magie d'un microscope, les pétales sont monumentalisés. Par cette intercession, l'instant et son image résistent au temps. Grâce aux procédés décrits (photographiques ou verriers), le vieillissement n'est plus à craindre. Les mediums sont garants de leur longévité. Ils saisissent le vif. Ils mémorisent et commémorent une existence éphémère.

\section{Bernard Moninot : I'agentivité végétale}

À la fin du printemps 1997, Bernard Moninot ${ }^{38}$ est dans son atelier du Pré-Saint-Gervais et ouvre les vasistas de la verrière, assez haute. Il est assis à sa table. Devant lui, dans un vase, une plante aquatique qu'il vient d'acheter, un Thalia, se diffuse en longues tiges verticales feuillées. Au sommet d'une de ces grandes tiges, une feuille, en forme d'amande, de lame au bout très pointu, bouge. Alors l'artiste commence à regarder le mouvement de la plante insufflé par un courant d'air. Il trouve ce phénomène très intéressant. «Il faudrait enregistrer ça » ${ }^{39}$ se dit-il. C'est la rencontre avec la première plante. C'est le point départ de la définition d'un dispositif pour Les Mémoires du Vent.

Il y eut tout un ensemble, un enchâssement d'idées constitutives des Mémoires du Vent. Parmi ces pistes, notamment, un intérêt fort et permanent de l'artiste pour les primitifs de la photographie invention majeure qui change la représentation du monde - et particulièrement Joseph Nicéphore Niepce $^{40}$, William Henry Fox Talbot ${ }^{41}$, Étienne-Jules Marey ${ }^{42}$, tous trois chercheurs et scientifiques.

Le premier est considéré comme l'inventeur précurseur de la photographie, sous le nom "héliographie", littéralement écriture par le soleil. Aussi N. Niepce rend-il possible, autour de 1825, la formation d'une image, sans l'intervention directe de l'homme, sans le recours de sa main. Cet intérêt inventif et manifeste se retrouve chez W. H. Fox Talbot, qui écrit dans « Remarques d'introduction » de son livre, au titre explicite, The Pencil of Nature/le crayon de la Nature, (1844) :

« Le petit ouvrage présenté au public est la première tentative de publier une série de planches ou d'images entièrement réalisées par le nouvel art du Dessin Photogénique, sans la moindre aide du crayon de l'artiste. ${ }^{43}$

\footnotetext{
${ }^{38}$ Bernard Moninot, artiste français né en 1949.

${ }^{39}$ Comme dans la partie précédente, les guillemets seuls de cette partie relèvent les propos de Bernard Moninot recueillis lors d'un entretien avec l'auteure le 13 janvier 2021.

40 Joseph « Nicéphore » Niepce (1765-1822), ingénieur - héliographie.

${ }^{41}$ William Henry Fox Talbot (1800-1877), scientifique britannique.

42 Étienne-Jules Marey (1830-1904), médecin et physiologiste français.

${ }^{43}$ W. H. F. Talbot, "Remarques d'introduction », The Pencil of Nature/Le crayon de la Nature, London, 1844, Partie 1, np. 


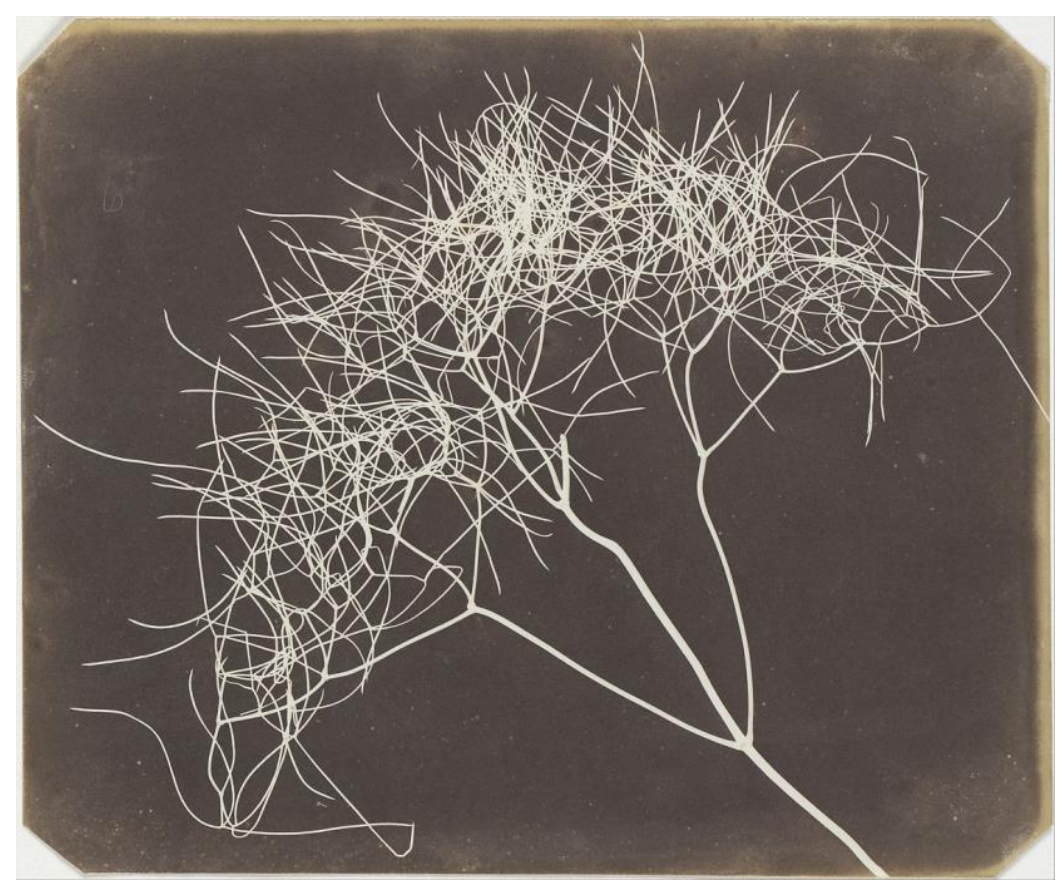

Figure 6. W. H. Fox Talbot, Fenouil sauvage, $1841^{44} 45$

$18,7 \times 22,7 \mathrm{~cm}$.

Ce qui est donné à voir tient à une écriture nullement retouchée. L’image, la transcription du réel, s'émancipe de la participation manuelle de l'artiste. Cela impliquera de nombreuses répercussions dans la conception des gestes artistiques en général ${ }^{46}$ et, près d'un siècle et demi plus tard aura de fortes incidences sur les créations de B. Moninot. Pour ce dernier, cela correspond à l'idée d'une nature capable de $\ll$ faire son propre portrait ${ }^{47}$.

Une autre recherche scientifique concourt à élaborer le dispositif. Sa mise au point est une résurgence, une évolution de ce que Bernard Moninot découvrait, il y a des années de cela, des recherches d'Étienne-Jules Marey en accédant aux archives du Musée de Beaune. «Des livres extraordinaires présentaient "sa méthode graphique" »: un petit capteur, que le médecin et physiologiste branchait sur le poignet d'un patient, transmettait et enregistrait ses battements de cœur sur un cylindre de laiton enduit de noir de fumée; ce diagramme graphique, relevant les pulsations, était en mesure de révéler les problèmes cardiaques du patient. Ainsi, grâce à cette expérience, est-il possible, une nouvelle fois, de déléguer à un processus l'élaboration d'une forme, d'une ligne et non pas de dessiner soi-même. Ainsi est transcrit quelque chose qui préexiste dans la

\footnotetext{
${ }^{44}$ W. H. Fox Talbot, Wild Fennel / Fenouil sauvage, impression sur papier salé, 1841-42, 18,7 x 22,7 cm, Metropolitan Museum of Art, New York.

45 À la même époque, l'amie de Talbot, la botaniste Anna Atkins (1799-1871), commence son Photographs of British Algae: Cyanotype Impressions en 1843 (petit tirage et exemplaires à diffusion privée, fabriqués un à un) Un des premiers livres scientifiques illustrés par la photographie.

${ }^{46}$ II y a là motif à un nécessaire déplacement de la pensée, de la « mission » et de l'intervention de l'artiste.

47 À noter : Talbot écrit aussi : "Pendant l'été 1835 je fis [...] un grand nombre de représentations de ma maison à la campagne [...]. Et je crois que ce bâtiment est le premier qui n'ait jamais été connu pour avoir dessiné sa propre image. ", "Some Account of the Art of Photogenic Drawing, or, the Process by Which Natural Objects May be Made to Delineate Themselves without the Aid of the Artist's Pencil (1839), repr. in Newhall, Photography ", in Newhall, Essays and Images, p. 28. II écrira ailleurs encore : "Ceci, je crois, est le premier exemple connu d'une maison ayant peint son propre portrait ", François Brunet, « Le crayon de la nature ", La naissance de l'idée de photographie, Paris, PUF, 2000 (2012), p. 133. 
nature, que l'on ne fait que dévoiler ${ }^{48}$. La manière est scientifique, elle vise à observer et transposer des phénomènes naturels sans user de la perception humaine.

Pour B. Moninot, quand on réfléchit à et avec des notions issues des sciences, on ne peut pas les rapporter de façon littérale, ce qui reviendrait à une simple citation. B. Moninot les déplace du territoire de la recherche (scientifique) au territoire de l'art, dans l'art. Pour lui, une invention, une découverte, doit passer par une phase d'appropriation, qui répond à des investigations autour d'une préoccupation singulière. Comment le vent dessine-t-il ? Comment la plante et le vent s'y prennentils pour dessiner? L'idée de Bernard Moninot est de capturer un dessin, une signature laissée par le vent avec la plante pour agent. Dans les Mémoires $d u$ vent, « est-ce la plante qui dessine ou c'est l'air?» s'interroge l'artiste. «C'est les deux : l'instrument qui trace, c'est la plante ; mais le vecteur c'est le vent. »

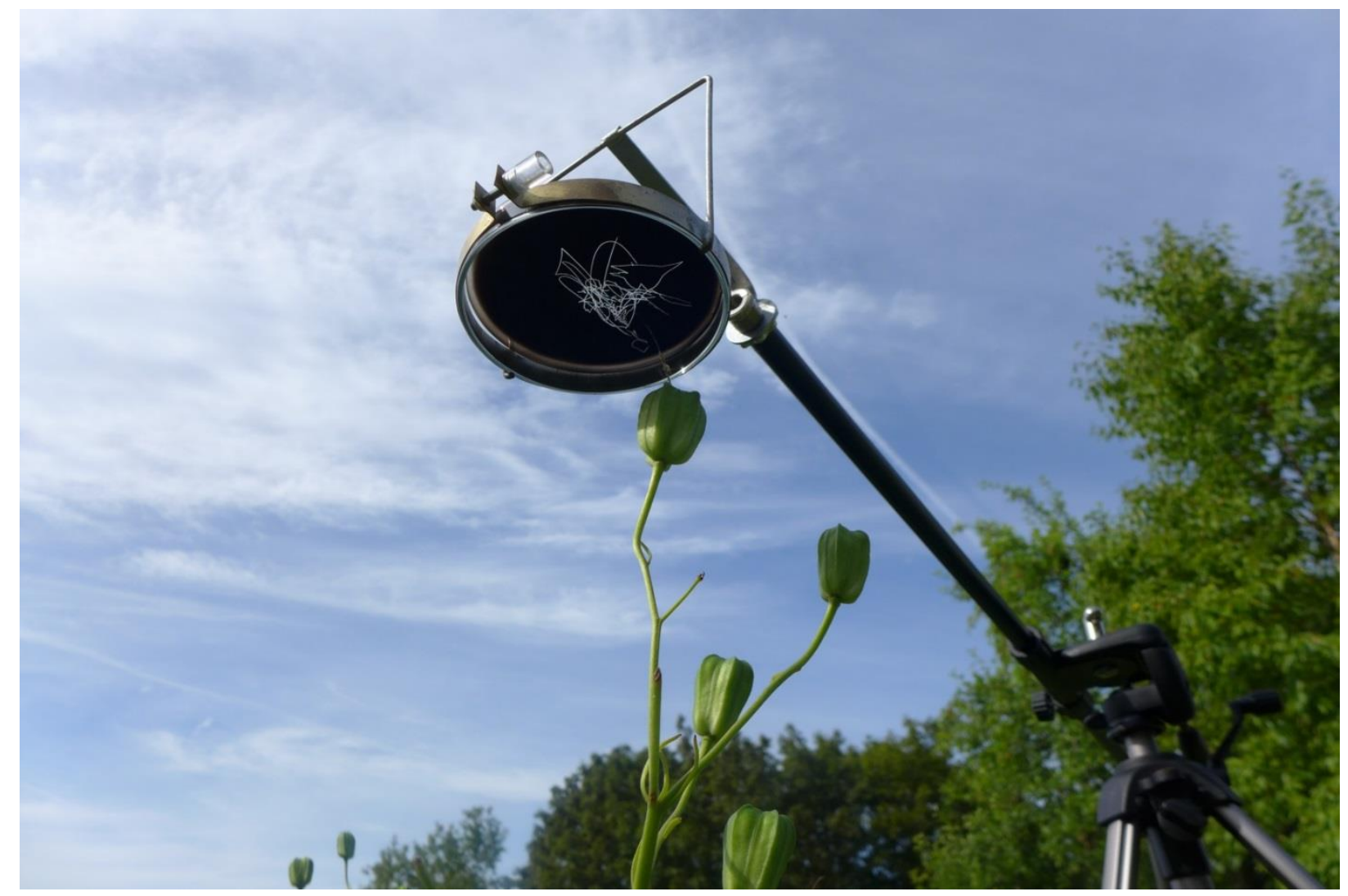

Figure 7. Mémoire du Vent - Captures au « Jardin de quatre vents » - la Malbaie

Québec - Canada - août 2015

(C) Bernard Moninot / ADAGP

\footnotetext{
48 Il est à noter que É.-J. Marey passa, à la fin de sa vie, trois années, de 1899 à 1901, à photographier les mouvements de l'air. Voir catalogue Mouvements de l'air, Étienne-Jules Marey, photographe des fluides, Gallimard/Réunion des Musées Nationaux, 2004, catalogue de l'exposition au Musée d'Orsay, 19 octobre 2004 - 16 janvier 2005.
} 


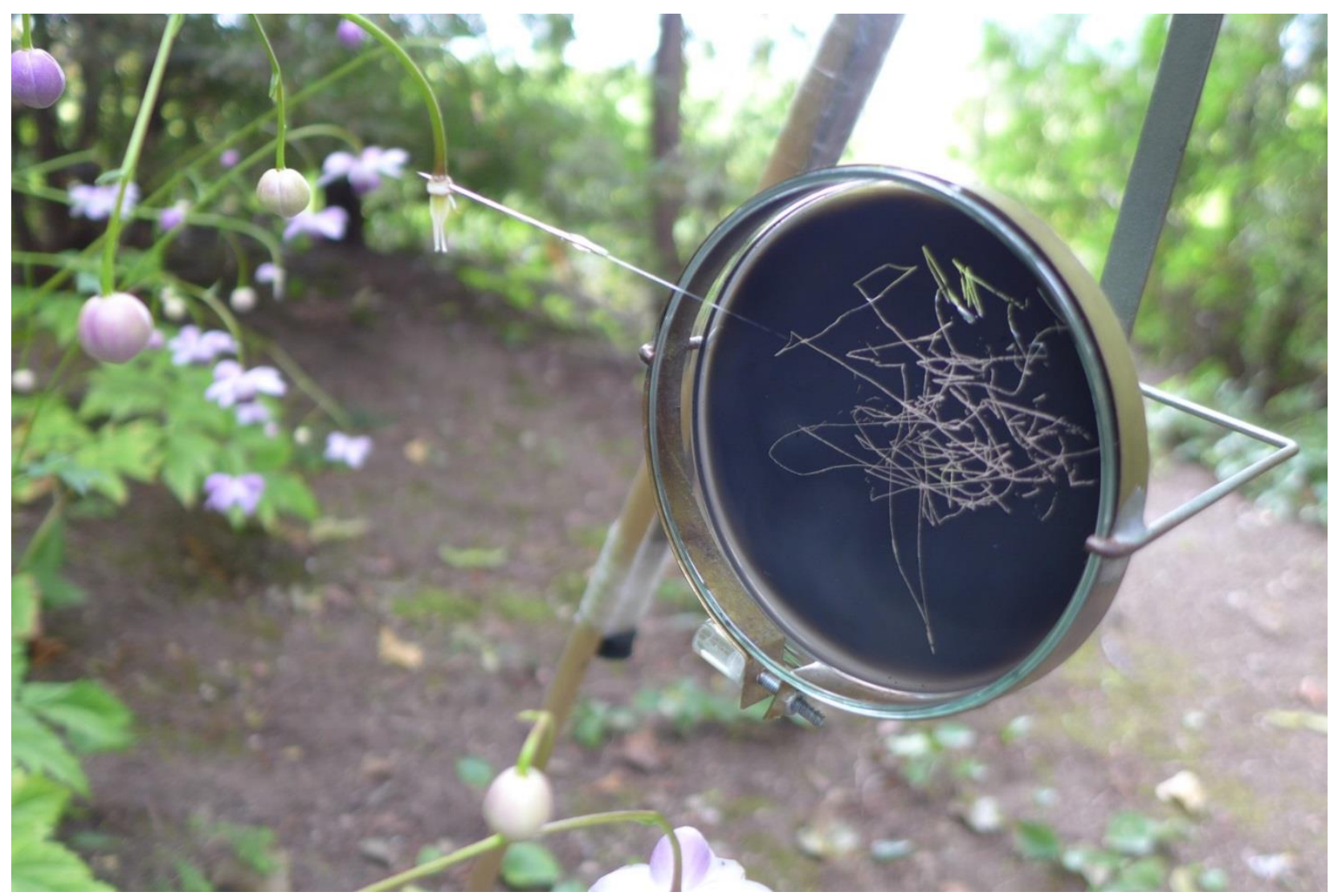

Figure 8. Mémoire du Vent - Captures au « Jardin de quatre vents » - la Malbaie

Québec - Canada - août 2015

(C) Bernard Moninot / ADAGP

L'artiste s'attache donc à la fabrication d'un instrument adéquat, qu'il décrit ainsi : " une fine aiguille de verre est collée à l'extrémité d'une branche ou d'une herbe qui oscille dans le vent, et grave, en quelques secondes un dessin unique sur la pellicule de carbone. La fonction de l'appareil est de recueillir cette écriture que le vent inscrit à l'intérieur de boîtes de Pétri enduites de noir de fumée $»^{49}$. Premier moment, le modus operandi est donné.

Un dispositif en plusieurs étapes : un protocole est établi, un ensemble d'opérations et de gestes effectués dans un certain ordre.

\section{La préparation :}

Une fois le dispositif pensé, tant d'un point de vue technique qu'idéel et la potence dotée d'une griffe qui maintiendra immobile l'appareil d'enregistrement réalisée, chaque «expédition» nécessite la préparation des boites de Pétri préalablement « enduites de noir de fumée » à l'intérieur et portant couvercles protecteurs, tout ceci contenu dans une sacoche pouvant contenir vingt boites, nombre modulaire des séries de la Mémoire du vent ${ }^{50}$.

\section{La destination - le moment, le lieu :}

La mécanique s'opère une première fois le 11 août 1999, dans les Cévennes, lors de la Grande éclipse du Soleil. Le processus débute. Il correspond à des instants où l'éclipse est totale, où le silence se fait absolu, où l'air s'arrête. Il n'y a plus de courant d'air. Pour B. Moninot, c'est « une relation entre la lumière du soleil et le vent, ce qui est poétiquement merveilleux ».

\footnotetext{
${ }^{49}$ Dans Jean-Christophe Bailly, Bernard Moninot, Marseille, André Dimanche éditeur, 2012, p. 276.

${ }^{50}$ Ensemble portatif permettant d'aller sans grande contrainte partout dans le monde. 
La méthode sera reconduite régulièrement, en France, en Inde, au Maroc, en Suisse, au Mexique, en Iran, au Canada, en des endroits signifiants. Le choix des lieux se fait principalement en ce qu'ils font sens, ce sont des lieux naturels exceptionnels, historiques ou personnels à l'artiste, jamais quelconques. Personnels à l'artiste comme les « reculés », sortes de canyons qui s'enfoncent dans le premier plateau jurassien, avec des falaises qui tombent sur cinquante mètres à pic, sur les bords desquelles poussent des plantes, dans la roche, presque perpendiculaires, au-dessus du vide, motivant souvent chez l'artiste « des appareillages que je faisais pour aller faire dessiner une plante qui survit dans des conditions "inimaginables" ». Des lieux avec des résonances historiques artistiques particulières, comme le Vent Paradis (Étival - Jura - lieu de villégiature en 1912 de Gabriële Buffet, Francis Picabia, Marcel Duchamp et Guillaume Apollinaire), des lieux botaniques exceptionnels parmi lesquels le Jardin botanique de Genève, le Jardin du Chellah ${ }^{51}$ au Maroc ou Fin Garden, en Iran, jardin persan clos de très hauts murs, abrité du vent, où une seule branche a dessiné l'ensemble de la séquence de la Mémoire du Vent.

\section{Le repérage - la sélection :}

Sur les lieux, l'artiste se promène, observe ${ }^{52}$. L'œil aux aguets, il s'agit pour lui de repérer, dans le paysage, les plantes qui vont servir pour enregistrer les mouvements de l'air ; aucun choix n'est anticipé, selon les variétés. L'essentiel est de regarder comment les végétaux bougent. Ce qui intéresse B. Moninot est relatif à un problème de dessinateur. L'artiste les voit dans l'espace ; il détaille les «aptitudes » des différentes parties - le sommet de la plante ou au contraire l'extrémité des branches, pétales et feuilles. Il faut que la plante présente un vrai potentiel à tracer et transmettre le vent dans l'apparition d'un dessin. Un exemple : sur sa terrasse à Château-Chalon, dans le Jura, poussent des roses trémières : "la rose trémière dessine extraordinairement bien » parce qu'elle est une plante très haute, assez solide et que son oscillation est très particulière $\left(40^{\prime}\right)$ : il enregistre celle de la partie supérieure de la plante. Le végétal peut être spécial ou commun, peu importe ; ce qui intéresse l'artiste, c'est de voir s'il est « doué ». Pour cela, il faut qu'il s'élève pour recevoir l'action du vent. L'étude du végétal (la botanique) se déplace ainsi vers de nouveaux critères, ceux ayant trait à sa mobilité.

\section{La capture - la collecte :}

L'artiste fiche, dans la plante, une aiguille, à la fois flexible et rigide, qui, par ses qualités, permet au dessin de se développer dans toutes les dimensions de l'espace. Alors il assiste à l'apparition du graphisme. Il y a là incorporation. B. Moninot dit : «L'incorporation est ressentie quand vous êtes vous-même dessinateur, quand vous découvrez quelque chose qui dessine à votre place, que vous êtes en train d'installer le dispositif et que vous attendez l'apparition d'une forme, qui s'élabore à l'instant même où vous la regardez, face à un phénomène qui ne dépend pas de vous. C'est tellement inattendu que cela produit directement un impact physique sur mon propre corps, avec une vigilance, avec une concentration telle que l'on s'arrête de respirer. Voilà quelque chose qui est produit par le vent. » L'opération se déroule sur une durée extrêmement brève, une récolte de quelques secondes, vingt s'il a beaucoup de vent, une minute dans d'autres cas. Il n'y a pas un temps régulier, extérieur, imposé au dessin. L'artiste regarde et, à un moment, en tension, décide que « ça suffit ».

\footnotetext{
51 Lieu emblématique où $B$. Moninot entreprend un travail spécifique sur le jardin entier. Pendant quinze jours, il collecte systématiquement 200 à 300 boites de verre.

${ }^{52}$ Voir citation JCB. 


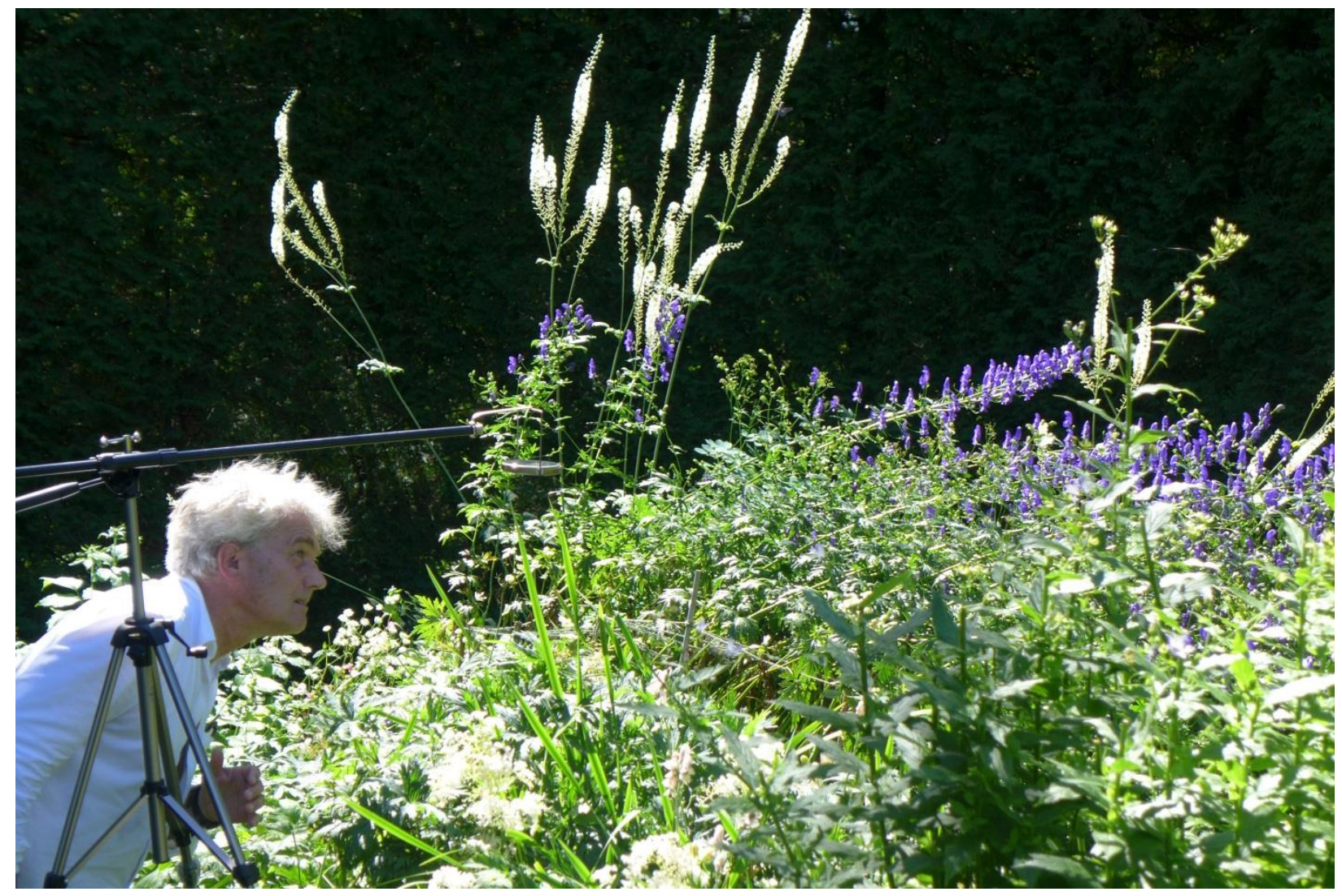

Figure 9. Bernard Moninot au « Jardin de quatre vents » - la Malbaie

Québec - Canada - août 2015

(C) Bernard Moninot / ADAGP

\section{L’arrêt - la décision - le dessin :}

Quand il a obtenu le bon dessin, de « haute-fidélité », très précis, avant qu'un endroit soit bouché, par le retour de la pointe sur ses propres pas, l'artiste déconnecte la plante du réceptacle de la trace, moment extrêmement décisif et délicat à la fois. À l'artiste revient la décision d'arrêter. Son souci, son intérêt majeur est le dessin, la qualité plastique littéralement de l'information qu'il récolte.

\section{Le devenir :}

D’abord l'artiste agit par intuition, et puis il analyse et réfléchit à comment poursuivre.

B. Moninot est prolixe dans les développements de ses processus. Ici, dans le texte se produit une ellipse temporelle, projetant vers une extension actuelle de la Mémoire du vent. Ces derniers temps, B. Moninot travaille à un nouveau projet dont il a déjà le titre, Le pin peint. Cet intitulé a une forte résonance ; effectivement, s'il s'inscrit dans une histoire relatée - celle de l'artiste visitant Man Ray qui, dans son atelier parisien, recouvre d'une peinture bleu monochrome une série de baguettes ${ }^{53}-$, il met en exergue le végétal, l'arbre « pin », se trouve énoncé et central dans ce nouveau dispositif. Sujet, dirait-on?

\footnotetext{
53 Man Ray (1890-1976), Pain peint : Nourriture préférée, des oiseaux bleus - 1958, baguette réelle peinte en bleu dont on connait deux exemplaires, par la suite éditée en résine, plâtre et bronze peints dans les années soixante. 


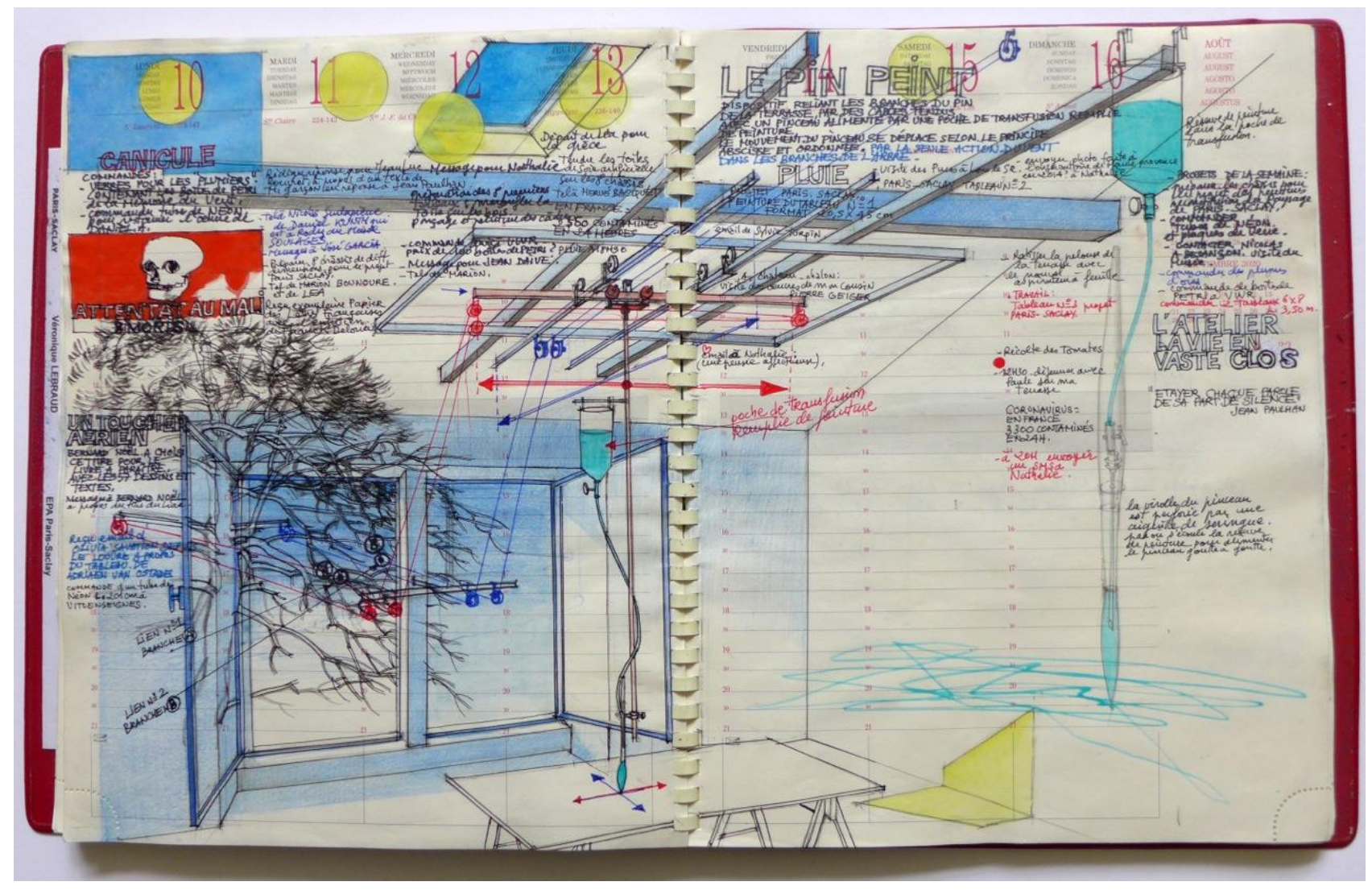

Figure 10. Bernard Moninot

dessin du dispositif "le Pin Peint" - page d'agenda, 2020

(c) Bernard Moninot / ADAGP

Aujourd'hui, sur la terrasse de B. Moninot, s'élance un arbre venant du plateau du Morvan. Planté durant son enfance, il faisait alors un mètre cinquante. C'était, il y a environ soixante ans, la durée de sa vie. L'artiste a dans l'idée d'intégrer son mouvement à une nouvelle pièce. Ce nouveau dessin implique la fabrication d'une machine, en collaboration avec un ami et assistant. Un système reliera les branches de l'arbre par des fils à un certain nombre d'instruments-relais installés dans son atelier, comme des poches de transfusion (de celles utilisées dans le milieu hospitalier). Celles-ci vont nourrir, en permanence, un pinceau suspendu qui se déplacera en fonction du tiraillement des filins. C'est là le principe du montage, perfectionné par l'usage d'un capteur électrique qui permettra des repérages selon le principe «abscisses - ordonnées ». Ainsi les différentes directions du pin animé par le vent seront transmises, transposées en courbes, en mouvements picturaux. L'heure est à la préparation des tableaux, aires de déplacement du pinceau sur des toiles carrées ${ }^{54}$ suivant les impulsions des branches de ce pin.

B. Moninot dit de ces travaux : «Je suis collecteur, les œuvres sont réalisées en co-production ; comme Gilles Clément ${ }^{55}$, je les cosigne avec la nature », déléguant l'apparition d'une forme, la part scripturale ou picturale de l'œuvre à un processus pensé à partir de et en collaboration avec les éléments de la nature. Que la nature fasse son propre portrait ${ }^{56}$. L'artiste aime à dire que ces dessins, ces peintures sont « acheiropoïètes », non faites de main d'homme.

Habituellement (ou classiquement), en représentant, l'artiste s'interpose et interprète le motif, par ses émotions, ses impressions; il y a une préoccupation dans l'œuvre de Moninot qui tend vers l'exactitude. "Le moment où va se rétablir le lien entre l'art et la science». "Qu'est-ce qu'un

\footnotetext{
${ }^{54}$ Toiles carrées, $1,50 \times 1,50 \mathrm{~m}$.

${ }^{55}$ Gilles Clément, né en 1943, paysagiste, jardinier, botaniste, entomologiste, biologiste et écrivain français.

${ }^{56}$ On rejoint là W. H. F. Talbot.

(c) 2021 ISTE OpenScience - Published by ISTE Ltd. London, UK - openscience.fr
} 
chercheur, en science ? Il ne parle pas de lui. Il met en place des protocoles pour récolter des informations, et élaborer des réflexions, une théorie, des choses qui vont transformer la façon dont nous percevons le monde. \ C'est cet axe-là, l'orientation principale de la recherche de l'artiste, que souligne Jean-Christophe Bailly quand il décèle dans le travail de Bernard Moninot une disposition : « [...] en direction des sciences de la nature, en direction de l'observation des phénomènes physiques [...] par ses références, ses matériaux mais davantage encore par son climat de recherche [...] ${ }^{57}$, par les champs de savoirs et de questionnements investis. Ici il est question de l'étude de processus de création. Regarder dans le réel ce qu'il y a de fertile pour son propre travail et entrer dans le processus. Ce n'est pas affaire d'imagination, mais vient principalement de l'observation de l'imagination qu'a la nature. «La nature, je n'ai pas commencé par cela. Plus j'avance, plus je suis fasciné, par ce que l'on voit, la capacité d'invention et surtout cet infini des possibles. »

Les mécanismes ici abordés impliquent le végétal dans sa dimension mobile et sensible, dans son agentivité, c'est-à-dire son potentiel à être un agent considéré selon sa capacité à intervenir et agir dans le monde - et non plus comme statique ou passif. Bernard Moninot n'aborde pas la plante isolément, il engage bien plus un regard sur l'environnement, tant spatial que temporel, tant géographique qu'historique. Il regarde ce qui se passe dans le sens où les éléments du milieu sont en connexion les uns aux autres, ce qui peut rappeler les conceptions mésologiques développées par Augustin Berque ${ }^{58}$.

\section{Conclusion}

Chez F. Morellet, le végétal est saisi pour révéler et questionner la forme dans son épure, chez A. et $\mathrm{P}$. Poirier pour présenter des qualités matérielles du vivant et de l'impermanence, et chez B. Moninot pour agir selon sa disposition remarquable à la mobilité. Pour chacun d'eux, il est un interlocuteur privilégié.

Ci-observés, les élans des artistes vers le végétal - prélevé en différents sens - le découvrent, loin des stratégies spéculatives. Ils engagent toute attention qui se démultiplie dans des sérialités nécessaires témoignant tout à la fois d'un approfondissement par l'expérimentation et d'une propension à la recherche suivant des systèmes inventés. Leurs intérêts se focalisent sur des manifestations discrètes de la nature, déplaçant l'idée souvent relayée que « l'homme attacherait du prix à ce qui $[. .$.$] aurait valeur de rareté { }^{59}$ et privilégierait les spécimens peu courants. La rareté se trouve ailleurs, dans l'expérience réitérée.

Ces artistes donnent à voir ce qui est potentiel, diffus et ténu, peut-être même invisible. Dans le même temps ils traduisent, dans leur contexte propre, la recommandation que formulait JeanJacques Rousseau à propos de la fleur dans son Dictionnaire des termes d'usage en botanique ${ }^{60}$. Au lieu de voir dans le végétal « une substance absolue », le considérer comme un "être collectif et relatif » en permanent échange avec son environnement, procédant par extension, physique et poétique, par correspondance inclusive et relationnelle.

\footnotetext{
57 Jean-Christophe Bailly, Bernard Moninot, « L'écoute (une pensée) », Marseille, André Dimanche, 2012, p. 12-13.

${ }^{58}$ Augustin Berque, Poétique de la Terre. Histoire naturelle et histoire humaine, essai de mésologie, Paris, Belin, 2014.

${ }^{59}$ Pensée de Eyama Masami mentionnée par Augustin Berque dans Le sauvage et l'artifice, Paris, Gallimard, 1986, p. 111.

60 Jean-Jacques Rousseau, Dictionnaire des termes d'usage en botanique, in Collection complète des œuvres, Genève, 17801789 , vol. 7, in-4 $4^{\circ}$, édition en ligne www.rousseauonline.ch, version du 7 octobre 2012, article « fleur ». 